\title{
Construction of asthma related competing endogenous RNA network revealed novel long non-coding RNAs and potential new drugs
}

Yifang Liao ${ }^{1+}$, Ping $\mathrm{Li}^{2+}$, Yanxia Wang ${ }^{3+}$, Hong Chen ${ }^{4^{*}+}$, Shangwei Ning ${ }^{3^{*+}}$ and Dongju Su ${ }^{4^{*}+}$ (D)

\begin{abstract}
Background: Asthma is a heterogeneous disease characterized by chronic airway inflammation. Long non-coding RNA can act as competing endogenous RNA to mRNA, and play significant role in many diseases. However, there is little known about the profiles of long non-coding RNA and the long non-coding RNA related competing endogenous RNA network in asthma. In current study, we aimed to explore the long non-coding RNA-microRNAmRNA competing endogenous RNA network in asthma and their potential implications for therapy and prognosis.

Methods: Asthma-related gene expression profiles were downloaded from the Gene Expression Omnibus database, re-annotated with these genes and identified for asthma-associated differentially expressed mRNAs and long noncoding RNAs. The long non-coding RNA-miRNA interaction data and mRNA-miRNA interaction data were downloaded using the starBase database to construct a long non-coding RNA-miRNA-mRNA global competing endogenous RNA network and extract asthma-related differentially expressed competing endogenous RNA network. Finally, functional enrichment analysis and drug repositioning of asthma-associated differentially expressed competing endogenous RNA networks were performed to further identify key long non-coding RNAs and potential therapeutics associated with asthma.

Results: This study constructed an asthma-associated competing endogenous RNA network, determined 5 key long non-coding RNAs (MALAT1, MIR17HG, CASC2, MAGI2-AS3, DAPK1-IT1) and identified 8 potential new drugs (Tamoxifen, Ruxolitinib, Tretinoin, Quercetin, Dasatinib, Levocarnitine, Niflumic Acid, Glyburide).

Conclusions: The results suggested that long non-coding RNA played an important role in asthma, and these novel long non-coding RNAs could be potential therapeutic target and prognostic biomarkers. At the same time, potential new drugs for asthma treatment have been discovered through drug repositioning techniques, providing a new direction for the treatment of asthma.
\end{abstract}

Keywords: Asthma, Long non-coding RNA, mRNA, Competing endogenous RNA network, Drug repositioning

\footnotetext{
*Correspondence: chenhong744563@aliyun.com;

ningsw@ems.hrbmu.edu.cn; hydhxsdj@126.com

${ }^{\dagger}$ All authors contributed equally to this work.

${ }^{3}$ College of Bioinformatics Science and Technology, Harbin Medical

University, Harbin, Heilongjiang, China

${ }^{4}$ Department of Respiratory Medicine, The 2nd Affiliated Hospital of Harbin

Medical University, Harbin, Heilongjiang, China

Full list of author information is available at the end of the article
}

(c) The Author(s). 2020 Open Access This article is distributed under the terms of the Creative Commons Attribution 4.0 International License (http://creativecommons.org/licenses/by/4.0/), which permits unrestricted use, distribution, and reproduction in any medium, provided you give appropriate credit to the original author(s) and the source, provide a link to the Creative Commons license, and indicate if changes were made. The Creative Commons Public Domain Dedication waiver (http://creativecommons.org/publicdomain/zero/1.0/) applies to the data made available in this article, unless otherwise stated. 


\section{Introduction}

Asthma is a chronic inflammatory disease of the airway which involves many cells and elements, resulting in airway hyperresponsiveness (AHR), excessive mucous secretion and expiratory airflow obstruction. Patients present with intermittent wheezing, chest tightness, shortness of breath and coughing triggered by infection, exercise, allergens or other stimuli. It is a serious public health problem around the world, affecting individuals of all age [1]. However, it has not been fully studied at the molecular level.

Long non-coding RNA (lncRNA) is a kind of noncoding RNA, with transcripts more than $200 \mathrm{bp}$ in length [2]. In recent years, IncRNA has gained widespread attention, as it can participate in a large range of biological processes, including regulation of apoptosis and invasion, reprogramming stem cell pluripotency, and parental imprinting [3, 4]. Previous studies have revealed some potential lncRNAs in asthma. For instance, a study uncovered that IncRNA TCF7 facilitated human airway smooth muscle cells (ASMCs) growth and migration by targeting TIMMDC1/Akt axis [5]. Meanwhile, Zhang et al. suggested that BCYRN1 may also regulate the proliferation and migration of ASMCs through upregulation of TRPC1 channel [6]. Another group revealed that lncRNA GAS5/miR-10a/BDNF regulatory axis contributed to the ASMCs proliferation [7]. In terms of asthma immunity, IncRNA MEG3 can regulate RORyt and affect Treg/Th17 balance via inhibiting miR-17 [8].

Competitive endogenous RNA (ceRNA) is a novel regulatory mechanism hypothesis: transcripts such as lncRNA, pseudogene transcripts or mRNA can be used as ceRNAs through microRNA (miRNA) response elements (MREs) to compete with miRNAs to regulate the expression level of the genes, thus affecting the function of the cells [9]. The ceRNA interactions have been found in respiratory diseases, especially in lung cancer. A study of non-small cell lung cancer (NSCLC) showed lncRNA LINC00702 could function as ceRNA for miR-510 to regulate PTEN expression, thus affected the proliferation and metastasis of cancer cells [10]. Another study found lncRNA NR2F2AS1 promoted NSCLC progression through regulating miR-320b/BMI1 axis [11]. In idiopathic pulmonary fibrosis (IPF), lncRNA PFAR regulated YAP1-Twist axis through targeting miR-138 as ceRNA, affected fibrogenesis in fibrotic lung [12]. The ceRNA mechanism for other respiratory diseases is constantly being explored, but its role in asthma is still unclear.

In this study, based on the ceRNA theory, we aimed to explore the regulatory IncRNA-miRNA-mRNA ceRNA network and key lncRNA in asthma by analyzing gene expression profile using bioinformatic methods. We downloaded the asthma-related gene expression profile (GSE43696) from the Gene Expression Omnibus (GEO) database, re-annotated these genes and identified asthmaspecific differentially expressed mRNAs, lncRNAs. We then constructed a IncRNA-miRNA-mRNA global ceRNA network and extracted asthma-related DE ceRNA network, from which we determined 5 key lncRNAs (MALAT1, MIR17HG, CASC2, MAGI2-AS3, DAPK1IT1). For further understanding of the key lncRNAs, we performed functional enrichment analysis. Additionally, drug repositioning was performed to discover new drug treatments for asthma, we identified 8 potential new drugs (Tamoxifen, Ruxolitinib, Tretinoin, Quercetin, Dasatinib, Levocarnitine, Niflumic Acid, Glyburide).

\section{Materials and methods \\ Gene expression profile}

We downloaded the asthma-related gene expression profile from the GEO database (http://www.ncbi.nlm. nih.gov/geo/) with the accession number of GSE43696 [13]. There were 108 samples (20 control and 88 cases). The mRNA sequence data and lncRNA sequence data were downloaded from the GENCODE (http://www.gencodegenes.org/) database. Approval from the Ethics Committee was not required for all the information was obtained from the GEO and GENCODE database.

\section{Probe re-annotation \\ Sequence alignment}

The mRNA sequence, the lncRNA sequence, and the probe sequence were sequence-aligned by the blastn tool, respectively. Basic Local Alignment Search Tool (BLAST) is a set of analysis tools for similarity comparison in protein databases or DNA databases which include blastn, blastp, blastx, tblastn, and tblastx. Blastn is a comparison of a given nucleic acid sequence to a sequence in a nucleic acid database. The length of each probe segment in the probe sequence information is 60 , so this data analysis selected the alignment result of the perfect match length of 60 .

\section{Probe screening}

According to the results of the sequence alignment, the probes which completely matched the lncRNA and the probes which completely matched the mRNA were separately screened. Probe screening relied mainly on the following two conditions: (1) The probe is only aligned to the mRNA sequence or only aligned to the lncRNA transcript sequence. (2) The probe is aligned to the only transcript sequence.

\section{Differential expression analysis}

Based on the mRNA-related expression profiles and lncRNA-related expression profiles, the significance analysis of microarrays (SAM) method [14] (q < 0.05, FDR) 
was used for differential expression analysis, performed using the SAM function in the R package samR [15].

\section{Network construction}

The IncRNA-miRNA interaction data and mRNAmiRNA interaction data were downloaded from the starBase V2.0 database (http://starbase.sysu.edu.cn/starbase2/index.php) [16], the lncRNA-miRNA-mRNA competing endogenous RNA network (global ceRNA network) was constructed by Cytoscape v3.5.1 software [17]. Based on the calculated differentially expressed mRNAs (DE mRNAs) and differentially expressed lncRNAs (DE lncRNAs), combined with global ceRNA network information, a subnetwork related to DE mRNA and DE lncRNA (DE ceRNA network) was extracted.

\section{Functional enrichment analysis}

The following databases were used as the source of function nodes: Gene Ontology (BP,MF,CC) (http://www.geneontology.org/) [18, 19], Kyoto Encyclopedia of Genes and Genomes (KEGG) pathway (https://www.genome.jp/kegg/ pathway.html) [20], REACTOME (pathway, reaction) (http://www.reactome.org) [21], Wiki Pathway (https:// www.wikipathways.org) [22], InterPro (http://www.ebi.ac. uk/interpro/) [23]. We performed functional enrichment analysis with the adjacent mRNAs of key lncRNAs by the $\mathrm{R}$ function 'phyper' $(p<0.05)$. And the 'pheatmap' function in R package was used for hierarchical clustering analysis, the relative expression levels were shown by the intensity of color.

\section{Drug repositioning}

We obtained the drug-gene targeting relationship and drug annotation information from the DrugBank database (https://www.drugbank.ca/) [24], and downloaded the drug-disease targeting relationship from the Therapeutic Target Database (TTD) (http://bidd.nus.edu.sg/ group/cjttd/) [25]. Then, we established a network of drugs and genes, we extracted asthma-related drug-gene interaction networks, integrated with DE ceRNA networks. In this way, we constructed a DE ceRNA-drug network and used Cytoscape v3.5.1 for visualization. To discover new asthma-targeted drugs, we integrated drug and disease targeting information and searched publications for drug repositioning.

\section{Results}

\section{Construction of global ceRNA network and DE ceRNA networks}

We downloaded the asthma related gene expression profile (GSE43696) from the GEO database, with 108 samples (20 control and 88 cases). The mRNA sequence data and lncRNA sequence data were downloaded from the GENCODE database. After downloading the data, we re-annotated the probes, identified DE mRNAs and DE IncRNAs with the method of SAM. As a result, a total of $266 \mathrm{DE}$ mRNAs and $31 \mathrm{DE}$ lncRNAs were identified.

In this study, IncRNA-miRNA interaction data and mRNA-miRNA interaction data were downloaded from the starBase V2.0 database to construct the global ceRNA network (Fig. 1a), visualization was performed with Cytoscape v3.5.1. There were 434,187 edges and 15, 317 nodes in the network, including 387 miRNAs, 1128 lncRNAs, 13,802 mRNAs, 10,212 miRNA-lncRNA interaction pairs (miRNA: 278, lncRNA: 1128), and 423,975 miRNA-mRNA interaction pairs (miRNA: 387, mRNA: 13802). Based on the calculated 266 DE mRNAs and 31 DE lncRNAs, combined with global ceRNA network information, DE ceRNA network was extracted (Fig. 1b). The DE ceRNA network contains 547 points and 6800 sides, including 378 miRNAs, 163 mRNAs, and 6 lncRNAs (MALAT1, MIR17HG, CASC2, MAGI2-AS3, DAPK1-IT1, CTD-3252C9.4). As the IncRNA CTD3252C9.4 just linked to 1 miRNA, we defined the remaining 5 lncRNAs as the key lncRNAs (MALAT1, MIR17HG, CASC2, MAGI2-AS3, DAPK1-IT1). For more details, we extracted each key IncRNA related ceRNA subnetwork (Fig. 1c).

\section{Literature validation of RNAs in key IncRNAs related ceRNA subnetworks}

We performed literature validation on IncRNAs, mRNAs, and miRNAs in key lncRNAs related ceRNA subnetworks to determine experimentally confirmed asthma-associated genes. There were 156 mRNAs, 44(28\%) have been reported to be associated with asthma, while $112(72 \%)$ of them were unverified (Fig. 2a). And we searched GeneCards database for asthma associated mRNAs, there was a score to each mRNA, the higher the score, the closer the mRNA is related to asthma. No study had assessed lncRNA MALAT1, MIR17HG, CASC2, MAGI2-AS3 and DAPK1-IT1 in asthma. As for miRNAs, only 5 miRNAs (hsa-miR-92a-3p, hsa-miR-93-5p, hsa-miR-126-5p, hsamiR-155-5p, hsa-miR-223-3p) have been verified. We linked the verified miRNAs to lncRNAs and mRNAs that connected to in the ceRNA subnetworks (Fig. 2b). And we considered that if a lncRNA linked to more literature validated miRNAs and mRNAs, it might be closer relevant to asthma.

Among the miRNAs, hsa-miR-92a-3p, hsa-miR-155$5 p$, and hsa-miR-223-3p were all linked to more than one lncRNA. The regulation of miR-92a-3p modified the transcription of KAT2B and PARP1, which played an important role in the development of combined allergic rhinitis and asthma syndrome [26]. Xiao et al. reported that miR-155 participated in immune regulation by setting a balance between Th1 and Th2 responses, and the 


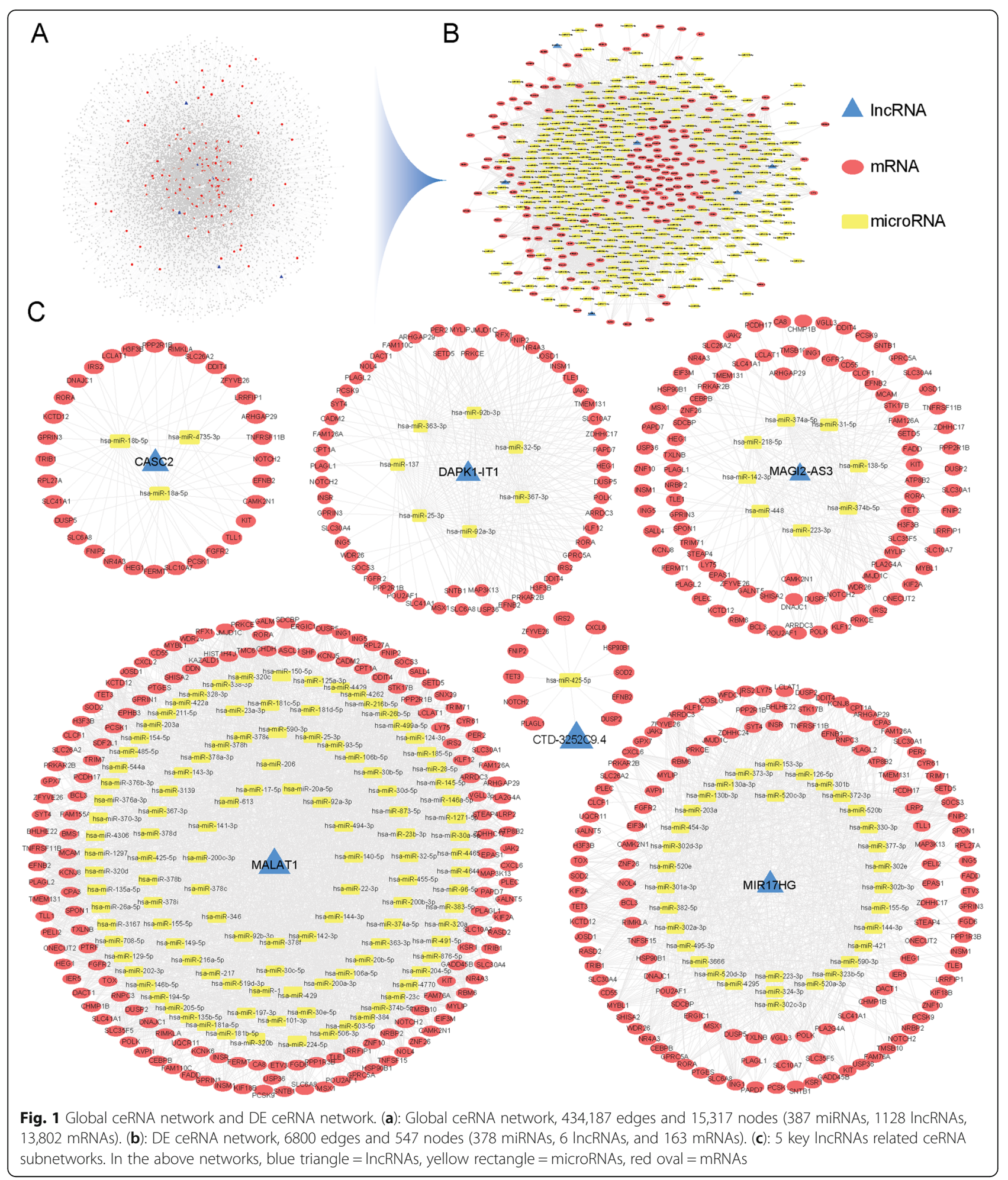

expression level of miR-155-5p was highly related to protein expression of the pro-asthmatic Th2 cytokines in bronchoalveolar lavage fluid (BALF) samples [27]. A study of sputum showed miR-223-3p was significantly upregulated in sputum of patients with severe asthma, and was highest in neutrophilic asthma, the expression was also associated with airway obstruction (FEV1/ forced vital capacity) [28].

CPA3 got the highest GeneCards score in mRNAs. Dougherty et al. demonstrated that CPA3 expression was 


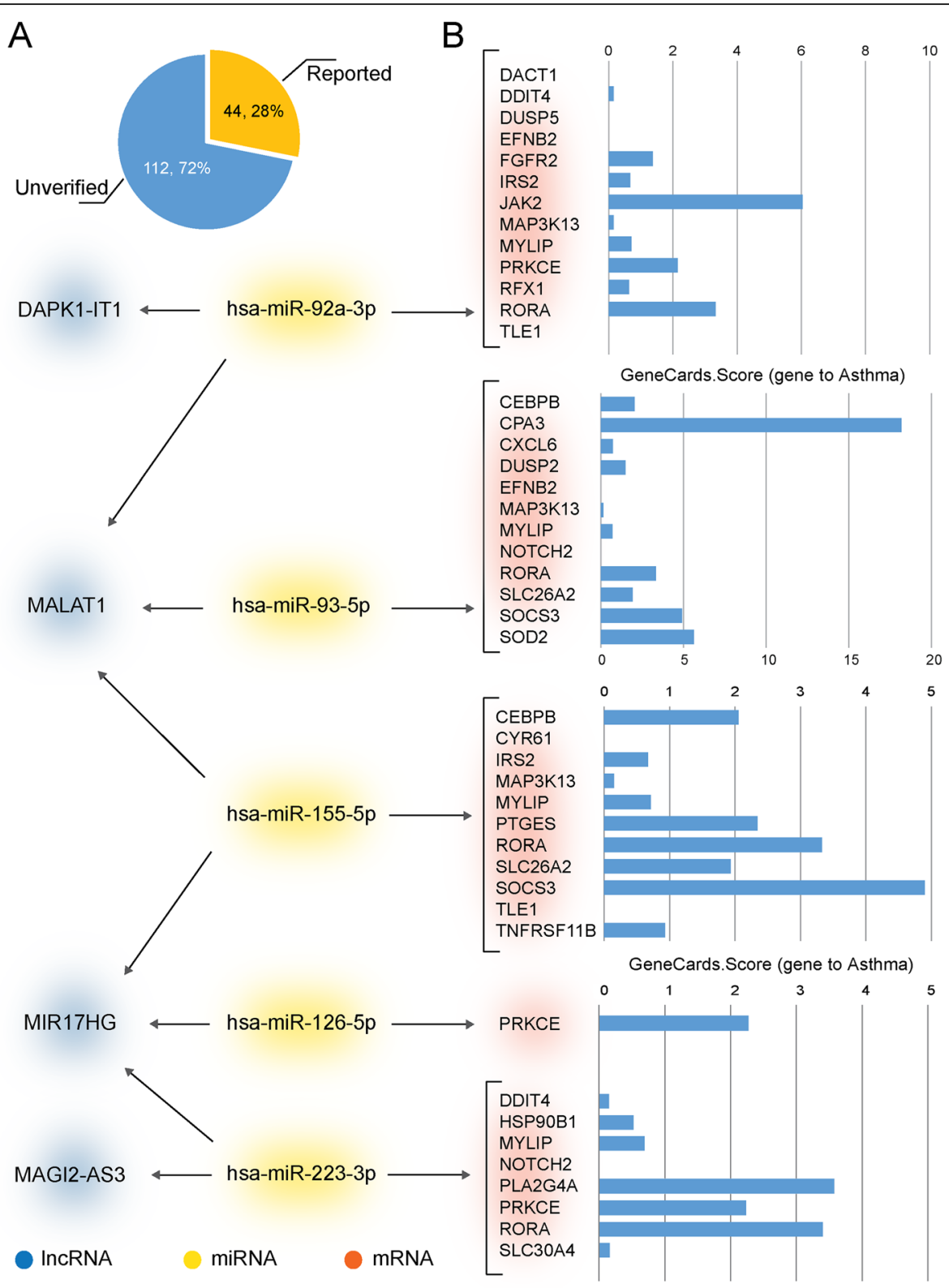

Fig. 2 Literature validation on IncRNA, mRNA, and miRNA in key IncRNA related ceRNA subnetworks. (a): A total of 156 mRNAs, 44(28\%) have been reported to be associated with asthma, 112(72\%) were unverified. (b): Verified miRNAs linked to the IncRNAs and mRNAs in the ceRNA subnetwork, $m R N A s$ were ranked with scores obtained from the GeneCards database. Blue nodes $=\operatorname{lncRNA}$, yellow nodes $=$ miRNA, red nodes $=m R N A$

especially increased in intraepithelial mast cells from subjects with TH2-high asthma, and it might play a role in asthma through production of angiotensin II [29]. By using the short interfering RNA (siRNA) technology, Zafra MP et al. found that after treated with SOCS3 siRNA, there were significant reduction of GATA3, Foxp3 gene expression and IL-10 mRNA production in eosinophils from asthmatics. In the meantime, SOCS3 siRNA treatment decreased the migration, adhesion and degranulation of eosinophil in response to IL-5. SOCS3 played an important regulatory role in asthma [30]. A metabolomic study of asthmatics indicated that PRKCE might be involved in the sphingolipid metabolism in asthma [31]. The JAK/STAT signaling pathway is an important cytokine signal transduction pathway. Ding F et al. discovered that LPS pretreatment inhibited IL-13 stimulation of ROS generation and the activation of JAK2/STAT6 pathways, thus promoted transcription factor FOXA2, inhibited excessive mucus secretion in asthma [32]. The other group showed miR-375 down regulated the expression of JAK2, inhibited the proliferation and migration of fetal ASM cells through regulating JAK2 / STAT3 signaling [33]. 
Functional enrichment analysis revealed key IncRNAs were closely related to asthma

To better understand the function of these key lncRNAs, functional enrichment analysis of the adjacent mRNAs of key lncRNAs were performed. The results were in eight aspects of the biological process (BP), molecular function (MF), cell composition (CC), KEGG pathways, REACTOME pathways, REACTOME reactions, Wiki pathways and InterPro protein domains (Fig. 3). Among them, MALAT1 and MIR17HG had the richest enrichment results and enriched in almost every aspect.

The functional analysis of MALAT1 revealed 106 GO terms (94 in BP, 1 in CC, 11 in MF), 8 KEGG pathways, 1 InterPro_ProteinDomains, 14 REACTOME_Pathways, 3 REACTOME_Reactions, and 11 Wiki_pathways. For MIR17HG, there were 94 GO terms (85 in BP, 9 in MF), 7 KEGG pathways, 1 InterPro_ProteinDomains, 13 REACTOME_Pathways, 2 REACTOME_Reactions, and 8 Wiki_ pathways. MALAT1 and MIR17HG had many common enrichment results that were important in asthma. Such as the regulation of STAT family, "transmembrane receptor protein kinase activity", "IL-17 signaling pathway", "Arachidonic acid metabolism", "Circadian Clock", "Interleukin-6 family signaling", etc. Moreover, MIR17HG had been involved in the $\mathrm{T}$ cell proliferation, and the component of sarcolemma, while MALAT1 been in TNF signaling pathway. In addition to the other three lncRNAs, CASC2: 6 GO terms (1 in BP, 5 in MF), 4 REACTOME_ Pathways, 2 REACTOME_Reactions. MAGI2-AS3: 24 GO terms (19 in BP, 5 in MF), 4 REACTOME_Pathways, 2 REACTOME_Reactions, and 3 Wiki_pathways. DAPK1IT1: 20 GO terms (17 in BP, 3 in MF), 3 KEGG pathways, 6 REACTOME_Pathways, and 4 Wiki_pathways. Even though CASC2, MAGI2-AS3, and DAPK1-IT1 had fewer results, they were also enriched in many asthma-related functions and pathways, as "cytokine production involved in immune response", "mesenchymal cell proliferation", "phosphatidylinositol 3-kinase activity", etc.

In the biological process, the phosphorylation of STAT took an important role. A study of asthmatic mice model showed after the antigen exposure, phosphorylation of STAT6 and STAT1 occurred at the early stage [34]. The inhibition of STAT1 effectively reduced allergen-induced airway inflammation and AHR [35]. Another study indicated that the activation of STAT3 was higher in mild asthma, and IL-6 stimulation increased STAT3 phosphorylation [36]. In the molecular function, 5 key lncRNAs were all enriched in "transmembrane receptor protein tyrosine kinase activity". The insulin-like growth factor-I receptor (IGF-IR) is a transmembrane heterotetrameric glycoprotein belonging to the transmembrane tyrosine kinase receptor family, it binds to IGF-I, IGF-II, and insulin [37]. Piñeiro-Hermida et al. investigated that after house dust mite (HDM) exposure, IGF-1R-deficient mice showed absent or attenuated AHR and airway inflammation, compared to control mice, indicated IG-1R as a potential therapeutic target for asthma [38]. IL-17 is an important cytokine in the inflammation of asthma. IL-17 receptor A (IL-17RA) is a receptor subunit of IL17A and IL-25. IL-17A enhanced airway constriction, and the blockage of IL-17RA, but not IL-17A, induced allergic inflammation and AHR. Therefore, IL-17A pathway can be stopped by blocking IL-17RA [39]. A study of mice showed pre-treatment of HDM allergens/PAR-2 increased IL-17A levels and IL-17RA expression, and the upregulation of IL-17A/IL-17RA deteriorated airway inflammation [40]. As the key lncRNAs involved in so many asthma-related functional results, we deduced that they might be relevant to asthma through gene regulation.

\section{Drug repositioning to discover new asthma-targeted drugs}

Drug repositioning is a strategy to find new indications for drugs which they are not originally intended, it is a faster and cheaper way to develop new therapies. By using the DrugBank and TTD database, we constructed a DE ceRNA-drug network (Fig. 4a), there were 46 drugs that might be the new asthma-targeted drugs. After searching literature in the Pubmed database, 8 drugs were pointed out, they were Tamoxifen, Ruxolitinib, Tretinoin, Quercetin, Dasatinib, Levocarnitine, Niflumic Acid, and Glyburide. In addition to Quercetin and Niflumic Acid which were still under experimenting, the other drugs have been approved by the FDA for disease treatment, and their targeted diseases were not asthma. The detailed information of these drugs was provided in Table 1. And drugs with potential pathways in asthma treatment are expressed in Fig. 5. LncRNA MIR17HG and MALAT1 were associated with all the above 8 drugs, we defined they were closely related with asthma, and extracted the ceRNA-drug subnetwork of MIR17HG and MALAT1 (Fig. $4 \mathrm{~b}$ and c).

Tamoxifen is one of the selective estrogen receptor modulators, which is mainly used to treat estrogen receptorpositive breast cancer. In vitro study showed that tamoxifen-induced neutrophil apoptosis and efferocytosis by alveolar macrophages, thus improved lung inflammation [41]. And another study of peripheral blood and BALF in horses indicated tamoxifen might induce apoptosis in granulocytic cells [42]. Ruxolitinib is a Janus-associated kinase inhibitor indicated to treat bone marrow cancer. In asthmatic mice model, the ruxolitinib intervention suppressed Th17 cells' survival through inhibiting JAK/STAT5 signaling pathway, promoted Th17 cells into apoptotic pathway by decreasing $\mathrm{Bcl}-2$ and increasing Caspase 3 expression [43]. Tretinoin, also known as all-trans-retinoic acid (ATRA), is used in the treatment of acute promyelocytic leukemia (APL). Wu et al. demonstrated ATRA decreased 


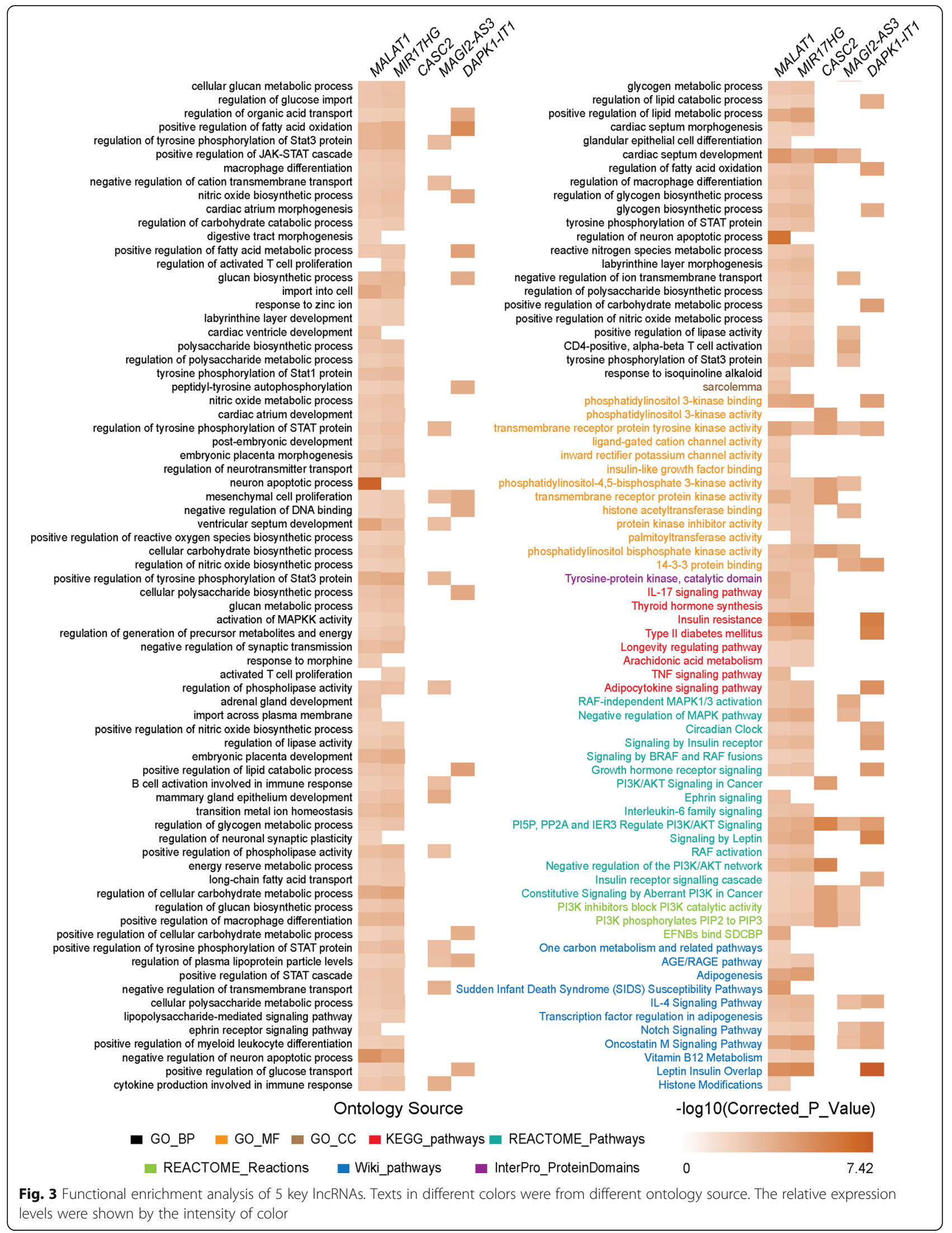




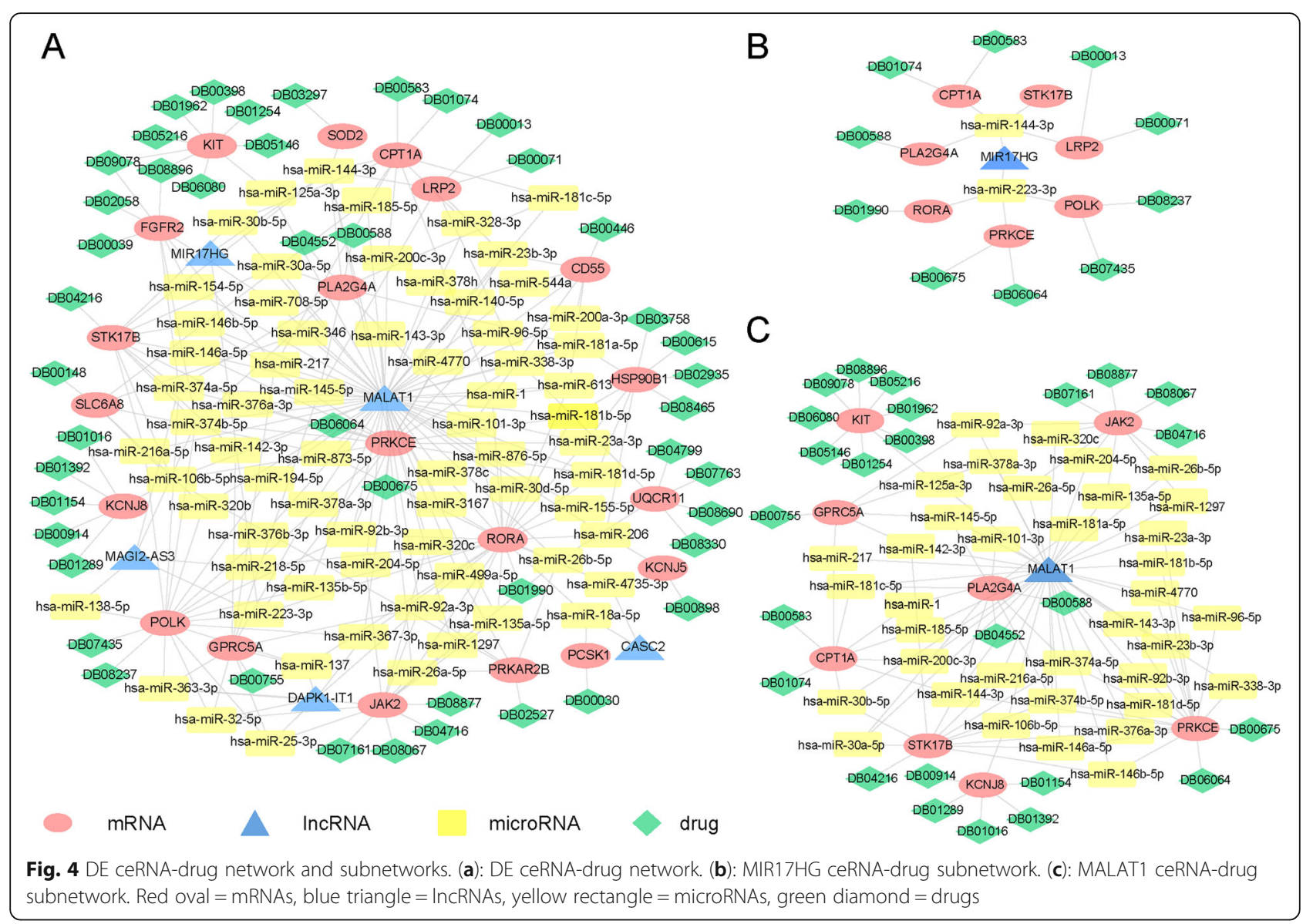

Th2 cytokines by inhibiting GATA-3 and increasing T-bet expression, also, ATRA down-regulated the expression of RORyt, thus decreased the level of IL-17A and the downstream proinflammatory factors [44]. Quercetin is a flavonol widely distributed in plants and is used to treat obesity. Pretreatment of quercetin was identified to inhibit gene expression of proinflammatory cytokine (TNF-a, IL$1 \mathrm{~b}, \mathrm{IL}-6$, and IL-8) by regulating the activation of NF- $\mathrm{kB}$ and p38 MAPK [45]. Dasatinib, Levocarnitine, Niflumic Acid and Glyburide were also been validated to be beneficial in asthma improvement [46-49]. On the other hand, the results of drug repositioning indicated that lncRNAs which linked to drug-associated mRNAs were closely related to asthma.

\section{Discussion}

Asthma is a heterogeneous disease, usually characterized by chronic airway inflammation. It is one of the most common chronic diseases and has been an economic burden all around the world [50]. In recent years, great efforts were done on discovering the new therapeutic targets and biomarkers of asthma, but most of the researches were focused on the protein-coding gene and small non-coding gene, the exploration of asthmarelated lncRNA is still in its infancy. For diseases like asthma that do not use pathology tests to diagnose, providing a molecular marker for early screening with body fluids will be a convenient diagnostic method. The lncRNA in blood, urine and other body fluids are stable and tissue-specific, making it the most suitable molecular marker for early diagnosis and prognosis monitoring of diseases.

During the present study, we identified differentially expressed lnRNAs, mRNAs according to data from GEO and GENCODE database. Then by utilizing bioinformatics tools, we constructed a IncRNA-miRNA-mRNA ceRNA network, from which we extracted asthma related DE ceRNA network and recognized 5 potential key lncRNAs (MALAT1, MIR17HG, CASC2, MAGI2-AS3 and DAPK1-IT1). Further more, we performed literature validation and functional enrichment analysis, which helped proved that these lncRNAs were closely related to asthma from the side aspect.

Among the 5 key lncRNAs, MALAT1 and MIR17HG were thought to be most crucial as they linked to more verified asthma-related miRNAs and mRNAs, also, the 
Table 1 Detailed information of the new asthma-targeted drugs

\begin{tabular}{|c|c|c|c|c|c|c|}
\hline $\begin{array}{l}\text { DrugBank } \\
\text { ID }\end{array}$ & Name & Gene & Drug Groups & Formula & Disease & $\begin{array}{l}\text { Gene Linked } \\
\text { IncRNA }\end{array}$ \\
\hline DB00675 & Tamoxifen & PRKCE & approved & $\mathrm{C} 26 \mathrm{H} 29 \mathrm{NO}$ & Breast cancer & $\begin{array}{l}\text { MIR17HG } \\
\text { MALAT1 } \\
\text { MAGI2-AS3 } \\
\text { DAPK1-IT1 }\end{array}$ \\
\hline DB08877 & Ruxolitinib & JAK2 & approved & $\mathrm{C} 17 \mathrm{H} 18 \mathrm{~N} 6$ & Essential thrombocythemia & $\begin{array}{l}\text { MIR17HG } \\
\text { MALAT1 } \\
\text { MAGI2-AS3 } \\
\text { DAPK1-IT1 }\end{array}$ \\
\hline DB00755 & Tretinoin & GPRC5A & $\begin{array}{l}\text { approved; investigational; } \\
\text { nutraceutical }\end{array}$ & $\mathrm{C} 2 \mathrm{OH} 28 \mathrm{O} 2$ & Leukemia & $\begin{array}{l}\text { MIR17HG } \\
\text { MALAT1 } \\
\text { MAGI2-AS3 } \\
\text { DAPK1-IT1 }\end{array}$ \\
\hline DB04216 & Quercetin & STK17B & experimental; investigational & $\mathrm{C} 15 \mathrm{H} 1007$ & Obesity & $\begin{array}{l}\text { MIR17HG } \\
\text { MALAT1 } \\
\text { MAGI2-AS3 }\end{array}$ \\
\hline DB01254 & Dasatinib & $\mathrm{KIT}$ & approved; investigational & $\mathrm{C} 22 \mathrm{H} 26 \mathrm{ClN} 7 \mathrm{O} 2 \mathrm{~S}$ & Mast cell leukemia & $\begin{array}{l}\text { MIR17HG } \\
\text { MALAT1 } \\
\text { MAGI2-AS3 } \\
\text { CASC2 }\end{array}$ \\
\hline DB00583 & Levocarnitine & CPT1A & approved; investigational & $\mathrm{C} 7 \mathrm{H} 15 \mathrm{NO} 3$ & Nutritional deficiency & $\begin{array}{l}\text { MIR17HG } \\
\text { MALAT1 } \\
\text { DAPK1-IT1 }\end{array}$ \\
\hline DB04552 & $\begin{array}{l}\text { Niflumic } \\
\text { Acid }\end{array}$ & PLA2G4A & experimental & $\mathrm{C} 13 \mathrm{H} 9 \mathrm{~F} 3 \mathrm{~N} 2 \mathrm{O} 2$ & $\begin{array}{l}\text { Rheumatoid arthritis; } \\
\text { Dysmenorrhea }\end{array}$ & $\begin{array}{l}\text { MIR17HG } \\
\text { MALAT1 } \\
\text { MAGI2-AS3 }\end{array}$ \\
\hline DB01016 & Glyburide & KCNJ8 & approved & $\mathrm{C} 23 \mathrm{H} 28 \mathrm{CIN} 3 \mathrm{O} 5 \mathrm{~S}$ & Type 2 diabetes & $\begin{array}{l}\text { MIR17HG } \\
\text { MALAT1 } \\
\text { MAGI2-AS3 }\end{array}$ \\
\hline
\end{tabular}

functional enrichment results were much abundant than the other 3 lncRNAs. Although MALAT1 is well-known as a tumor-associated lncRNA [51], it also involved in inflammatory progression. Recently, more and more studies had explored its function in inflammatory progression. A study reported that MALAT1 regulated polarization of macrophage and response to lung injury [52]. Another study revealed MALAT1 regulated T-cell differentiation towards a Tregs phenotype, played an anti-inflammatory role in autoimmune neuroinflammation [53]. MIR17HG or the miR-17/92 cluster host gene was identified in 2004 [54]. The miR-17/92 cluster consists of six miRNAs: miR-17, miR-18a, miR-19a, miR-19b-1, miR-20a, and miR-92a-1. Carraro et al. had found that the miR-17/92 cluster was involved in normal lung morphogenesis, epithelial proliferation, and branching by targeting STAT3 and MAPK14 [55]. And the components of mir-17/92 cluster were also found to play roles in asthma. In a study of human bronchial epithelial cells (BEC), the expression of miR-19a was upregulated in severe asthmatics, which enhanced cell proliferation of BEC by targeting TGF- $\beta$ receptor 2 , the repression of miR-19a expression increased SMAD3 and decreased BEC proliferation [56].
For IncRNA CASC2, MAGI2-AS3 and DAPK1-IT1, probably because of the lack of current study, resulting in fewer analytical results, but they still have great potential to explore.

Clinically, the most effective way to treat and prevent exacerbation of asthma is drug therapy. At present, asthma drugs are mainly divided into two parts, bronchodilators and anti-inflammatory drugs. Although the current drugs are effective in treating most asthma patients, there is still a part of patients to whom the drugs are not working. In search of new drugs for asthma treatment, we performed drug repositioning by using the data obtained from DrugBank and TTD. And we got 8 drugs: Tamoxifen, Ruxolitinib, Tretinoin, Quercetin, Dasatinib, Levocarnitine, Niflumic Acid, and Glyburide. Although these drugs have been reported to be effectively improve asthma related symptoms and inflammatory process, there is still a distance from clinical application, further research should be done regarding drug dosage, administration methods, and dosage related side effects, etc.

\section{Conclusions}

In summary, by constructing asthma related ceRNA network and conducting bioinformatic analysis, we 


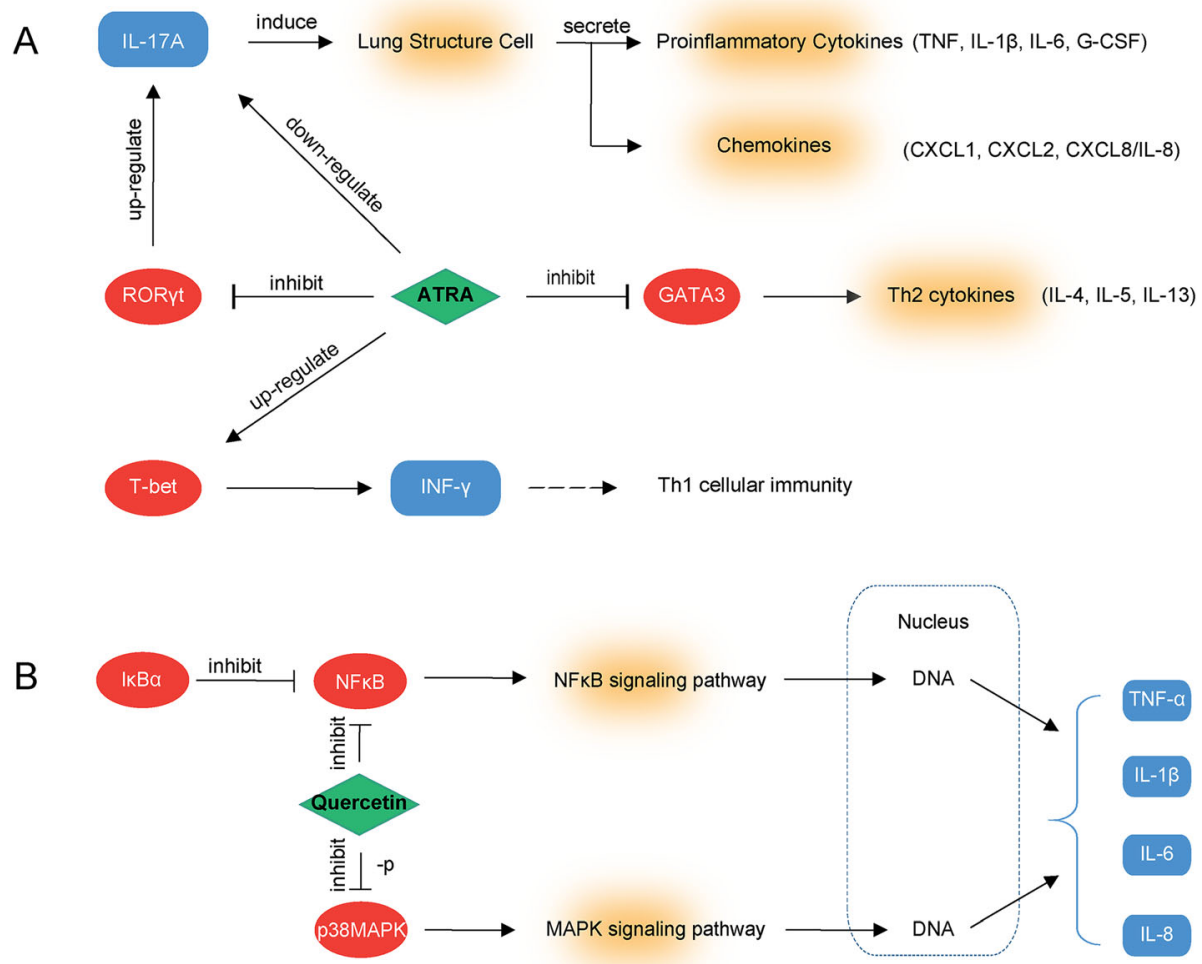

Fig. 5 The potential mechanism of drugs in asthma. (a): The potential mechanism of Tretinoin (ATRA) in asthma. ATRA decreased Th2 cytokines by inhibiting GATA-3 and up-regulating T-bet expression, also, ATRA inhibited the expression of RORyt, thus decreased the level of IL-17A and the downstream proinflammatory factors. (b): The potential mechanism of Quercetin in asthma. Quercetin inhibited gene expression of proinflammatory cytokine (TNF-a, IL-1b, IL-6, and IL-8) by regulating the activation of NF-KB and p38 MAPK

identified 5 asthma associated key lncRNAs (MALAT1, MIR17HG, CASC2, MAGI2-AS3, DAPK1-IT1) and 8 potential new drugs (Tamoxifen, Ruxolitinib, Tretinoin, Quercetin, Dasatinib, Levocarnitine, Niflumic Acid, Glyburide). The current findings provide novel insights into the new therapeutic target and prognostic biomarker for asthma, and future work is needed to validate the key lncRNAs and their function in the ceRNA network.

\section{Abbreviations}

AHR: Airway hyperresponsiveness; ASMCs: Airway smooth muscle cells; ATRA: All-trans-retinoic acid; BALF: Bronchoalveolar lavage fluid; BEC: Bronchial epithelial cells; BLAST: Basic Local Alignment Search Tool; BP: Biological process; CC: Cell composition; ceRNA: Competing endogenous RNA; DE ceRNA network: Subnetwork related to DE mRNA and DE IncRNA; DE IncRNAs: Differentially expressed IncRNAs; DE mRNAs: Differentially expressed mRNAs; GEO: Gene Expression Omnibus; HDM: House dust mite; IGF-IR: Insulin-like growth factor-I receptor; IL-17RA: IL-17 receptor A; IPF: Idiopathic pulmonary fibrosis; KEGG: Kyoto Encyclopedia of Genes and Genomes; IncRNA: Long non-coding RNA; MF: Molecular function; miRNA: MicroRNA; MREs: MiRNA response elements; NSCLC: Non-small cell lung cancer; SAM: Significance analysis of microarrays; siRNA: Short interfering RNA; TTD: Therapeutic Target Database

\section{Acknowledgments}

Not applicable.
Authors' contributions

$D J, H C$, and SW designed the study and revised the manuscript; YF, PL, and $Y X$ carried out literature research, performed the experiments and wrote this article. All the authors read and approved the final manuscript.

\section{Funding}

No grant funding to report.

\section{Availability of data and materials}

The dataset used and/or analyzed during the current study is available in the GEO repository, https://www.doi.org.10.1038/mi.2014.6 [13].

Ethics approval and consent to participate

Not applicable.

\section{Consent for publication}

Not applicable.

\section{Competing interests}

No conflicts of interest, financial or otherwise, are declared by the authors.

\section{Author details}

${ }^{1}$ Department of Respiratory Medicine, The 2nd Affiliated Hospital of Xiamen Medical College, Xiamen, Fujian, China. ${ }^{2}$ Department of Radiology, The 2nd Affiliated Hospital of Harbin Medical University, Harbin, Heilongjiang, China. ${ }^{3}$ College of Bioinformatics Science and Technology, Harbin Medical University, Harbin, Heilongjiang, China. ${ }^{4}$ Department of Respiratory Medicine, The 2nd Affiliated Hospital of Harbin Medical University, Harbin, Heilongjiang, China. 
Received: 21 August 2019 Accepted: 6 December 2019

\section{Published online: 10 January 2020}

\section{References}

1. Loftus PA, Wise SK. Epidemiology of asthma. Curr Opin Otolaryngol Head Neck Surg. 2016;24(3):245-9.

2. Mercer TR, Dinger ME, Mattick JS. Long non-coding RNAs: insights into functions. Nat Rev Genet. 2009;10(3):155-9.

3. Batista PJ, Chang HY. Long noncoding RNAs: cellular address codes in development and disease. Cell. 2013;152(6):1298-307.

4. Lee JT, Bartolomei MS. X-inactivation, imprinting, and long noncoding RNAs in health and disease. Cell. 2013;152(6):1308-23.

5. Fan M, Xu J, Xiao Q, Chen F, Han X. Long non-coding RNA TCF7 contributes to the growth and migration of airway smooth muscle cells in asthma through targeting TIMMDC1/Akt axis. Biochem Biophys Res Commun. 2019; 508(3):749-55

6. Zhang XY, Zhang LX, Tian CJ, Tang XY, Zhao LM, Guo YL, et al. LncRNAs BCYRN1 promoted the proliferation and migration of rat airway smooth muscle cells in asthma via upregulating the expression of transient receptor potential 1. Am J Transl Res. 2016;8(8):3409-18.

7. Zhang XY, Tang XY, Li N, Zhao LM, Guo YL, Li XS, et al. GAS5 promotes airway smooth muscle cell proliferation in asthma via controlling miR-10a/ BDNF signaling pathway. Life Sci. 2018;212:93-101.

8. Qiu YY, Wu Y, Lin MJ, Bian T, Xiao YL, Qin C. LncRNA-MEG3 functions as a competing endogenous RNA to regulate Treg/Th17 balance in patients with asthma by targeting microRNA-17/ RORgammat. Biomed Pharmacother. 2018; 111:386-94.

9. Salmena $L$, Poliseno L, Tay $Y$, Kats $L$, Pandolfi PP. A ceRNA hypothesis: the Rosetta stone of a hidden RNA language? Cell. 2011;146(3):353-8.

10. Yu W, Li D, Ding X, Sun Y, Liu Y, Cong J, et al. LINC00702 suppresses proliferation and invasion in non-small cell lung cancer through regulating miR-510/PTEN axis. Aging (Albany NY). 2019;11(5):1471-85.

11. Zhang S, Zhang X, Sun Q, Zhuang C, Li G, Sun L, et al. LncRNA NR2F2-AS1 promotes tumourigenesis through modulating BMI1 expression by targeting miR-320b in non-small cell lung cancer. J Cell Mol Med. 2019; 23(3):2001-11

12. Zhao X, Sun J, Chen Y, Su W, Shan H, Li Y, et al. IncRNA PFAR promotes lung fibroblast activation and fibrosis by targeting miR-138 to regulate the YAP1-twist Axis. Mol Ther. 2018;26(9):2206-17.

13. Voraphani N, Gladwin MT, Contreras AU, Kaminski N, Tedrow JR, Milosevic J, et al. An airway epithelial iNOS-DUOX2-thyroid peroxidase metabolome drives Th1/Th2 nitrative stress in human severe asthma. Mucosal Immunol. 2014;7(5):1175-85.

14. Tusher VG, Tibshirani R, Chu G. Significance analysis of microarrays applied to the ionizing radiation response. Proc Natl Acad Sci U S A. 2001;98(9): $5116-21$.

15. Tan H, Tian Y, Yang H, Liu G, Nie L. A novel Streptomyces gene, samR, with different effects on differentiation of Streptomyces ansochromogenes and Streptomyces coelicolor. Arch Microbiol. 2002;177(3):274-8.

16. Li JH, Liu S, Zhou H, Qu LH, Yang JH. starBase v2.0: decoding miRNAceRNA, miRNA-ncRNA and protein-RNA interaction networks from largescale CLIP-Seq data. Nucleic Acids Res. 2014;42(Database issue):D92-7.

17. Shannon P, Markiel A, Ozier O, Baliga NS, Wang JT, Ramage D, et al. Cytoscape: a software environment for integrated models of biomolecular interaction networks. Genome Res. 2003;13(11):2498-504.

18. Ashburner M, Ball CA, Blake JA, Botstein D, Butler H, Cherry JM, et al. Gene ontology: tool for the unification of biology. The Gene Ontology Consortium. Nat Genet. 2000;25(1):25-9.

19. Consortium TGO. Expansion of the gene ontology knowledgebase and resources. Nucleic Acids Res. 2017:45(D1):D331-D8.

20. Kanehisa M, Furumichi M, Tanabe M, Sato Y, Morishima K. KEGG: new perspectives on genomes, pathways, diseases and drugs. Nucleic Acids Res. 2017:45(D1):D353-D61.

21. Fabregat A, Jupe S, Matthews L, Sidiropoulos K, Gillespie M, Garapati P, et al The Reactome pathway knowledgebase. Nucleic Acids Res. 2018;46(D1): D649-D55.

22. Slenter DN, Kutmon M, Hanspers K, Riutta A, Windsor J, Nunes N, et al. WikiPathways: a multifaceted pathway database bridging metabolomics to other omics research. Nucleic Acids Res. 2018:46(D1): D661-d7.
23. Mitchell AL, Attwood TK, Babbitt PC, Blum M, Bork P, Bridge A, et al. InterPro in 2019: improving coverage, classification and access to protein sequence annotations. Nucleic Acids Res. 2019;47(D1):D351-D60.

24. Wishart DS, Feunang YD, Guo AC, Lo EJ, Marcu A, Grant JR, et al. DrugBank 5.0: a major update to the DrugBank database for 2018. Nucleic Acids Res. 2018;46(D1):D1074-D82.

25. Li YH, Yu CY, Li XX, Zhang P, Tang J, Yang Q, et al. Therapeutic target database update 2018: enriched resource for facilitating bench-to-clinic research of targeted therapeutics. Nucleic Acids Res. 2018;46(D1):D1121-d7.

26. Mao Z, Shi Y, Cao Q, Chen Y, Sun Y, Liu Z, et al. Transcriptional regulation on the gene expression signature in combined allergic rhinitis and asthma syndrome. Epigenomics. 2018;10(2):119-31.

27. Xiao R, Noel A, Perveen Z, Penn AL. In utero exposure to second-hand smoke activates pro-asthmatic and oncogenic miRNAs in adult asthmatic mice. Environ Mol Mutagen. 2016:57(3):190-9.

28. Maes T, Cobos FA, Schleich F, Sorbello V, Henket M, De Preter K, et al. Asthma inflammatory phenotypes show differential microRNA expression in sputum. J Allergy Clin Immunol. 2016;137(5):1433-46.

29. Dougherty RH, Sidhu SS, Raman K, Solon M, Solberg OD, Caughey GH, et al. Accumulation of intraepithelial mast cells with a unique protease phenotype in $\mathrm{T}(\mathrm{H}) 2$-high asthma. J Allergy Clin Immunol. 2010;125(5): 1046-53.e8.

30. Zafra MP, Canas JA, Mazzeo C, Gamez C, Sanz V, Fernandez-Nieto M, et al. SOCS3 silencing attenuates eosinophil functions in asthma patients. Int J Mol Sci. 2015;16(3):5434-51.

31. McGeachie MJ, Dahlin A, Qiu W, Croteau-Chonka DC, Savage J, Wu AC, et al. The metabolomics of asthma control: a promising link between genetics and disease. Immun Inflamm Dis. 2015:3(3):224-38.

32. Ding F, Liu B, Zou W, Tian D, Li Q, Dai J, et al. LPS exposure in early life protects against mucus Hypersecretion in ovalbumin-induced asthma by Down-regulation of the IL-13 and JAK-STAT6 pathways. Cell Physiol Biochem. 2018;46(3):1263-74.

33. Ji Y, Yang $X$, Su H. Overexpression of microRNA-375 impedes plateletderived growth factor-induced proliferation and migration of human fetal airway smooth muscle cells by targeting Janus kinase 2. Biomed Pharmacother. 2018;98:69-75.

34. Chiba Y, Todoroki M, Misawa M. Antigen exposure causes activations of signal transducer and activator of transcription 6 (STAT6) and STAT1, but not STAT3, in lungs of sensitized mice. Immunopharmacol Immunotoxicol. 2011;33(1):43-8.

35. Quarcoo D, Weixler S, Groneberg D, Joachim R, Ahrens B, Wagner AH, et al. Inhibition of signal transducer and activator of transcription 1 attenuates allergen-induced airway inflammation and hyperreactivity. J Allergy Clin Immunol. 2004;114(2):288-95.

36. Salem $\mathrm{H}$, Plante $S$, Gounni AS, Rouabhia $M$, Chakir J. A shift in the IL6/STAT3 signalling pathway imbalance towards the SHP2 pathway in severe asthma results in reduced proliferation process. Cell Signal. 2018:43:47-54.

37. Lee H, Kim SR, Oh Y, Cho SH, Schleimer RP, Lee YC. Targeting insulin-like growth factor-I and insulin-like growth factor-binding protein-3 signaling pathways. A novel therapeutic approach for asthma. Am J Respir Cell Mol Biol. 2014;50(4):667-77.

38. Pineiro-Hermida S, Gregory JA, Lopez IP, Torrens R, Ruiz-Martinez C, Adner $M$, et al. Attenuated airway hyperresponsiveness and mucus secretion in HDM-exposed Igf1r-deficient mice. Allergy. 2017;72(9):1317-26.

39. Willis CR, Siegel $L$, Leith $A$, Mohn D, Escobar S, Wannberg $S$, et al. IL-17RA signaling in airway inflammation and bronchial Hyperreactivity in allergic asthma. Am J Respir Cell Mol Biol. 2015;53(6):810-21.

40. Nadeem A, Al-Harbi NO, Ahmad SF, Ibrahim KE, Alotaibi MR, Siddiqui N, et al. Protease activated receptor-2 mediated upregulation of IL-17 receptor signaling on airway epithelial cells is responsible for neutrophilic infiltration during acute exposure of house dust mite allergens in mice. Chem Biol Interact. 2019;304:52-60.

41. Olave C, Morales N, Uberti B, Henriquez C, Sarmiento J, Ortloff A, et al. Tamoxifen induces apoptotic neutrophil efferocytosis in horses. Vet Res Commun. 2018:42(1):57-63.

42. Sarmiento J, Perez B, Morales N, Henriquez C, Vidal L, Folch H, et al. Apoptotic effects of tamoxifen on leukocytes from horse peripheral blood and bronchoalveolar lavage fluid. Vet Res Commun. 2013;37(4):333-8.

43. Li RF, Wang GF. JAK/STAT5 signaling pathway inhibitor ruxolitinib reduces airway inflammation of neutrophilic asthma in mice model. Eur Rev Med Pharmacol Sci. 2018;22(3):835-43. 
44. Wu J, Zhang Y, Liu Q, Zhong W, Xia Z. All-trans retinoic acid attenuates airway inflammation by inhibiting Th2 and Th17 response in experimental allergic asthma. BMC Immunol. 2013;14:28.

45. Min YD, Choi CH, Bark H, Son HY, Park HH, Lee S, et al. Quercetin inhibits expression of inflammatory cytokines through attenuation of NF-kappaB and p38 MAPK in HMC-1 human mast cell line. Inflamm Res, 2007;56(5):210-5.

46. da Silva AL, Magalhaes RF, Branco VC, Silva JD, Cruz FF, Marques PS, et al. The tyrosine kinase inhibitor dasatinib reduces lung inflammation and remodelling in experimental allergic asthma. Br J Pharmacol. 2016;173(7): 1236-47.

47. Al-Biltagi M, Isa M, Bediwy AS, Helaly N, El Lebedy DD. L-carnitine improves the asthma control in children with moderate persistent asthma. J Allergy (Cairo). 2012;2012:509730.

48. Kondo M, Nakata J, Arai N, Izumo T, Tagaya E, Takeyama K, et al. Niflumic acid inhibits goblet cell degranulation in a Guinea pig asthma model. Allergol Int. 2012;61(1):133-42.

49. Cui W, Zhang S, Cai Z, Hu X, Zhang R, Wang Y, et al. The antidiabetic agent glibenclamide protects airway hyperresponsiveness and inflammation in mice. Inflammation. 2015;38(2):835-45.

50. Loftus PA, Wise SK. Epidemiology and economic burden of asthma. Int Forum Allergy Rhinol. 2015;5(Suppl 1):S7-10.

51. Li ZX, Zhu QN, Zhang HB, Hu Y, Wang G, Zhu YS. MALAT1: a potential biomarker in cancer. Cancer Manag Res. 2018;10:6757-68.

52. Cui H, Banerjee S, Guo S, Xie N, Ge J, Jiang D, et al. Long noncoding RNA Malat1 regulates differential activation of macrophage and response to lung injury. JCl Insight. 2019;4(4):e124522.

53. Masoumi F, Ghorbani S, Talebi F, Branton WG, Rajaei S, Power C, et al. Malat1 long noncoding RNA regulates inflammation and leukocyte differentiation in experimental autoimmune encephalomyelitis. J Neuroimmunol. 2018:328:50-9.

54. Ota A, Tagawa H, Karnan S, Tsuzuki S, Karpas A, Kira S, et al. Identification and characterization of a novel gene, C13orf25, as a target for 13q31-q32 amplification in malignant lymphoma. Cancer Res. 2004;64(9):3087-95.

55. Carraro G, El-Hashash A, Guidolin D, Tiozzo C, Turcatel G, Young BM, et al. miR-17 family of microRNAs controls FGF10-mediated embryonic lung epithelial branching morphogenesis through MAPK14 and STAT3 regulation of E-cadherin distribution. Dev Biol. 2009;333(2):238-50.

56. Haj-Salem I, Fakhfakh R, Berube JC, Jacques E, Plante S, Simard MJ, et al. MicroRNA-19a enhances proliferation of bronchial epithelial cells by targeting TGFbetaR2 gene in severe asthma. Allergy. 2015;70(2):212-9.

\section{Publisher's Note}

Springer Nature remains neutral with regard to jurisdictional claims in published maps and institutional affiliations.

Ready to submit your research? Choose BMC and benefit from:

- fast, convenient online submission

- thorough peer review by experienced researchers in your field

- rapid publication on acceptance

- support for research data, including large and complex data types

- gold Open Access which fosters wider collaboration and increased citations

- maximum visibility for your research: over $100 \mathrm{M}$ website views per year

At $\mathrm{BMC}$, research is always in progress.

Learn more biomedcentral.com/submissions 